\title{
RILEM TC 247-DTA round robin test: mix design and reproducibility of compressive strength of alkali- activated concretes
}

\author{
John L. Provis $($ ) Kamel Arbi - Susan A. Bernal • Dali Bondar • \\ Anja Buchwald · Arnaud Castel · Sundararaman Chithiraputhiran • \\ Martin Cyr - Alireza Dehghan - Katja Dombrowski-Daube - Ashish Dubey • \\ Vilma Ducman • Gregor J. G. Gluth • Sreejith Nanukuttan • Karl Peterson • \\ Francisca Puertas • Arie van Riessen - Manuel Torres-Carrasco • \\ Guang Ye $\cdot$ Yibing Zuo
}

Received: 5 May 2019/ Accepted: 26 August 2019/Published online: 10 September 2019

(C) The Author(s) 2019

\begin{abstract}
The aim of RILEM TC 247-DTA 'Durability Testing of Alkali-Activated Materials' is to identify and validate methodologies for testing the durability of alkali-activated concretes. To underpin the durability testing work of this committee, five alkali-activated concrete mixes were developed based on blast furnace slag, fly ash, and flash-calcined metakaolin. The concretes were designed with
\end{abstract}

This article was prepared within the framework of RILEM TC 247-DTA. The article has been reviewed and approved by all members of the TC.

TC Membership

Chair: John L. Provis, UK

Deputy Chair: Frank Winnefeld, Switzerland

TC Members: Kamel Arbi, Netherlands; P.A. Muhammed Basheer, UK; Susan A. Bernal, UK; Dali Bondar, UK; Lorenza Carabba, Italy; Arnaud Castel, Australia; Maria Chiara Bignozzi, Italy; Anja Buchwald, Netherlands; Huisu Chen, P.R. China; Sundaraman Chithiraputhiran, USA; Francesco Colangelo, Italy; Andrzej Cwirzen, Sweden; Martin Cyr, France; Alireza Dehghan, Canada; Katja Dombrowski-Daube, Germany; Ashish Dubey, USA; Vilma Ducman, Slovenia; Ana Fernández-Jiménez, Spain; Ellis M. Gartner, France; Gregor J.G. Gluth, Germany; R. Douglas Hooton, Canada; Kazuo Ichimiya, Japan; Elie Kamseu, Cameroon; Lesley S.-C. Ko, USA; Sabina Kramar, Slovenia; Pavlo V. Kryvenko, Ukraine; Yuwei Ma, P.R. China; Isabel Martins, Portugal; Sreejith V. Nanukuttan, UK; Angel Palomo, Spain; Karl Peterson, Canada; different intended performance levels, aiming to assess the capability of test methods to discriminate between concretes on this basis. A total of fifteen laboratories worldwide participated in this round robin test programme, where all concretes were produced with the same mix designs, from single-source aluminosilicate precursors and locally available aggregates. This paper reports the mix designs tested, and the

Penny Pipilikaki, Netherlands; Francisca Puertas, Spain; Aljoša Šajna, Slovenia; Jay G. Sanjayan, Australia; Caijun Shi, P.R. China; Marios N. Soutsos, UK; Dietmar Stephan, Germany; Arezki Tagnit-Hamou, Canada; Monique Tognonvi, Canada; Manuel Torres, Spain; Siska L.A. Valcke, Netherlands; Arie van Riessen, Australia; Jannie S.J. van Deventer, Australia; Jeanette H.M. Visser, Netherlands; Steffen Wache, Germany; Claire E. White, USA; Guang Ye, Netherlands; Yibing Zuo, Netherlands.

J. L. Provis $(\bowtie) \cdot$ S. A. Bernal

Department of Materials Science and Engineering, The

University of Sheffield, Sheffield S1 3JD, UK

e-mail: j.provis@sheffield.ac.uk

K. Arbi · G. Ye · Y. Zuo

Microlab, Faculty of Civil Engineering and Geosciences, Delft University of Technology, Stevinweg 1,

2628 CN Delft, The Netherlands

K. Arbi

Delta Concrete Consult B. V., Marconiweg 2,

4131 PD Vianen, The Netherlands 
compressive strength results obtained, including critical insight into reasons for the observed variability in strength within and between laboratories.

Keywords Alkali-activated concretes .

Compressive strength - Blast furnace slag · Fly ash ·

Metakaolin $\cdot$ Round robin

\section{Introduction}

To enable the full-scale concrete production using innovative binding systems, including (but not limited to) alkali-activated materials [1], it is essential that standardisation of concrete moves from a prescriptive basis toward a performance-based philosophy [2]. This will bring the necessary flexibility for material producers to develop a formulation that is suited to the particular application at hand, using all available materials in the most efficient and effective way possible.

However, performance-based specification of concretes depends fundamentally on the assumption that valid performance tests are available-and this is a significant challenge when considering durability testing of non-Portland cement-based concretes, for which the accelerated testing methods that are commonly

\section{S. A. Bernal}

School of Civil Engineering, University of Leeds, Leeds LS2 9JT, United Kingdom

D. Bondar $\cdot$ S. Nanukuttan

School of Natural and Built Environment, Queen's University Belfast, Belfast BT9 5AG, UK

A. Buchwald

ASCEM B.V., Beek, The Netherlands

\section{A. Castel}

School of Civil and Environmental Engineering, University of New South Wales, Kensington, NSW 2052, Australia

S. Chithiraputhiran · A. Dubey

USG Corporation, Libertyville, IL, USA

M. Cyr

Laboratoire Matériaux et Durabilité des Constructions (LMDC), Université de Toulouse, Toulouse Cedex 04, France applied to Portland cement-based materials are not necessarily an accurate representation of the degradation that would take place in the field. This was discussed in detail by RILEM TC 224-AAM 'AlkaliActivated Materials' [1], and the outcomes of that committee included identification of the need to conduct inter-laboratory validation of existing standardised tests, to assess whether they can be used to gain meaningful information related to the durability of alkali-activated concretes. To this end, RILEM TC 247-DTA was established, with the remit to develop and implement a round-robin assessment of whether it is valid to test alkali-activated concretes according to the protocols of testing methods that are widely used for the assessment of Portland cement-based concretes.

The underpinning philosophy of TC 247-DTA is therefore based on "testing the tests". There was no intention to develop a highly optimised alkali-activated concrete mix based on a particular precursor or activator, or to provide general statements about the suitability of these concretes for use under any particular set of conditions. Rather, five concretes of varying expected performance levels were designed and produced, using three widely available precursors, with the aim of applying tests to discriminate between these different performance levels in a laboratory context. This study design is based on the concept that,

\author{
A. Dehghan · K. Peterson \\ Department of Civil and Mineral Engineering, University \\ of Toronto, 35 St. George St., Toronto, ON M5S 1A4, \\ Canada \\ K. Dombrowski-Daube \\ Technical University Bergakademie Freiberg, Freiberg, \\ Germany \\ V. Ducman \\ ZAG Ljubljana, Dimičeva 12, 1000 Ljubljana, Slovenia
}

G. J. G. Gluth

Bundesanstalt für Materialforschung und -prüfung (BAM), Unter den Eichen 87, 12205 Berlin, Germany

F. Puertas · M. Torres-Carrasco

Eduardo Torroja Institute for Construction Sciences (IETcc-CSIC), Madrid, Spain

A. van Riessen

John de Laeter Research Centre, Curtin University, Perth, WA 6845, Australia 
if all materials within a testing campaign provide a high performance level, it is impossible to know whether the testing methods could actually identify a material that is not performing at acceptable levels, as this would have been outside the scope of the validation of the testing method. The mix designs were kept intentionally as simple as possible, to ensure that issues around the use of proprietary additives (intellectual property, worldwide availability, reproducibility) were kept to a minimum. Single-batch sources of ground granulated blast furnace slag, fly ash, and flash-calcined metakaolin were obtained and distributed to all participants of the testing programme, although locally-available aggregates were used in each laboratory as the cost of transporting such quantities of material would have proven prohibitive for a global testing programme.

This paper reports the mix designs selected for use throughout the work of RILEM TC 247-DTA, and focuses mainly on the assessment of the compressive strength of the concretes produced, including in particular discussion of the causes of variation within and between laboratories.

\section{Experimental methodology}

\subsection{Materials}

The key precursors used in all testing laboratories were: ground granulated blast furnace slag (GBFS) supplied by Ecocem (France), siliceous fly ash (FA) supplied by BauMineral (Germany), and flash-calcined metakaolin (MK) supplied by Argeco (France). Each material was sourced from the supplier initially as a single batch distributed to all participants, and the results reported here refer to the single-batch materials, although some later work on durability testing required sourcing by some participants of additional material which had been batched separately. Differences between precursor batches were found to be minimal in terms of material composition, mineralogy and reactivity. The chemical compositions of all materials are shown in Table 1, and their basic physical properties are listed in Table 2.

The amorphous fraction of the fly ash was determined by Rietveld analysis with recorded X-ray diffraction patterns to be $74.9 \mathrm{wt} \%$; the crystalline phases were mullite, quartz, ferrite spinel phase(s), hematite and periclase, and minor amounts of anhydrite, calcite and lime. The GBFS was almost completely amorphous, containing only minor amounts of

Table 1 Chemical composition of precursors used for concrete production, as determined by X-ray fluorescence (XRF; shown in plain text) or inductively coupled plasma optical emission

spectroscopy (ICP-OES) after total digestion (shown in italics); LOI is the loss on ignition, measured in air at either 950 or $1000{ }^{\circ} \mathrm{C}$

\begin{tabular}{lccccccccccc}
\hline Precursor & $\mathrm{CaO}$ & $\mathrm{SiO}_{2}$ & $\mathrm{Al}_{2} \mathrm{O}_{3}$ & $\mathrm{MgO}$ & $\mathrm{SO}_{3}$ & $\mathrm{Fe}_{2} \mathrm{O}_{3}$ & $\mathrm{TiO}_{2}$ & $\mathrm{~K}_{2} \mathrm{O}$ & $\mathrm{Na}_{2} \mathrm{O}$ & $\mathrm{LOI}$ \\
\hline GBFS & 43.9 & 35.7 & 11.2 & 6.5 & 0.4 & 0.3 & 0.5 & 0.4 & 0.2 & 0.3 \\
& 41.4 & 36.3 & 11.3 & 6.4 & 1.1 & 0.4 & 0.5 & 0.4 & 0.3 & 0.0 \\
& 41.8 & 36.0 & 11.3 & 6.5 & 0.7 & 0.3 & 0.5 & 0.4 & 0.1 & 1.9 \\
& 43.6 & 36.5 & 10.7 & 6.0 & 1.1 & 0.4 & 0.5 & 0.4 & 0.3 & 0.1 \\
FA & 6.0 & 53.1 & 22.1 & 2.4 & 0.6 & 8.0 & 0.9 & 2.2 & 0.9 & 2.4 \\
& 7.3 & 48.6 & 21.7 & 2.4 & 0.8 & 8.0 & 1.0 & 2.1 & 1.1 & 5.3 \\
& 6.0 & 51.8 & 23.0 & 2.5 & 0.5 & 7.4 & 0.9 & 2.2 & 0.8 & 2.3 \\
& 5.6 & 53.5 & 21.9 & 2.5 & 0.5 & 8.3 & 1.0 & 2.2 & 1.0 & 2.4 \\
MK & 0.5 & 68.8 & 24.3 & 0.2 & - & 2.3 & 1.1 & 0.2 & - & 1.6 \\
& 0.9 & 68.1 & 24.1 & 0.2 & 0.03 & 3.7 & 1.1 & 0.4 & 0.1 & - \\
& 0.7 & 72.0 & 22.0 & 0.1 & $<0.04$ & 2.2 & 1.1 & 0.2 & 0.1 & 1.4 \\
\hline
\end{tabular}

All elements are represented on an oxide basis; compositions do not sum to $100 \%$ as some of the elements present in incompletelyoxidised form contribute to both oxide and (negative) LOI constituents, and some minor constituents (e.g. MnO, BaO) are not shown. Each row shows the determination conducted in a particular laboratory, and the differences between them provide an indication of the expected uncertainty in this type of XRF analysis of SCMs 
Table 2 Physical properties of precursors used for AAM concrete production

\begin{tabular}{llcl}
\hline Precursor & $d_{50}(\mu \mathrm{m})^{\mathrm{a}}$ & BET surface area $\left(\mathrm{cm}^{2} / \mathrm{g}\right)$ & Density $\left(\mathrm{kg} / \mathrm{m}^{3}\right)$ \\
\hline GBFS & 11.8 & 995 & 2880 \\
& & 1070 & 2360 \\
FA & 12.8 & 1700 & 2200 \\
MK & - & 16,280 & \\
\hline
\end{tabular}

${ }^{a}$ Determined by laser diffraction using a dry dispersion unit; at least four measurements were taken for each precursor, with standard deviation $0.1 \mu \mathrm{m}$

gehlenite and calcite. The metakaolin contained a considerable amount of quartz as well as minor quantities of other crystalline impurities.

Each laboratory formulated sodium silicate activators, of identical composition according to the mix design protocols provided to participants, by blending locally available commercial sodium silicate solutions of modulus (molar ratio $\mathrm{SiO}_{2} / \mathrm{Na}_{2} \mathrm{O}$ ) of approximately 2.0, with appropriate quantities of reagent-grade $\mathrm{NaOH}$ and tap water, to reach the specified modulus for each concrete mix. Some laboratories employed sodium silicates of different compositions, e.g. modulus 1.68, since such solutions could be used as the sole activator constituent to produce the metakaolinbased concrete mix. For logistical reasons, each laboratory used their locally available aggregates; some used siliceous aggregates and some calcareous, and each used the closest possible approximation to the specified grading curves based on local materials availability and established practice.

Table 3 Mix design parameters of the alkali-activated concretes tested

\begin{tabular}{|c|c|c|c|c|c|c|c|c|}
\hline Concrete & $\begin{array}{l}\text { Precursor } \\
\left(\mathrm{kg} / \mathrm{m}^{3}\right)\end{array}$ & $\begin{array}{l}\text { Sodium } \\
\text { silicate } \\
\text { dose }^{\mathrm{a}}\end{array}$ & $\begin{array}{l}\text { Sodium } \\
\text { hydroxide } \\
\text { dose }^{b}\end{array}$ & $\begin{array}{l}\text { Water/ } \\
\text { binder } \\
\text { mass ratio }^{c}\end{array}$ & 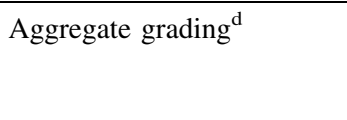 & $\begin{array}{l}\text { Estimated } \\
\text { air content } \\
(\%)^{\mathrm{e}}\end{array}$ & $\begin{array}{l}\text { Design } \\
\text { density } \\
\left(\mathrm{kg} / \mathrm{m}^{3}\right)^{\mathrm{e}}\end{array}$ & Notes \\
\hline S3a & $\begin{array}{c}\text { GBFS, } \\
375\end{array}$ & 2.69 & 4 & 0.382 & $\begin{array}{l}40 \% \text { sand } 0-4 \mathrm{~mm}, 60 \% \\
\text { gravel } 4-16 \mathrm{~mm} \text {, to meet } \\
\text { A/B } 16 \text { curve }\end{array}$ & 1.0 & 2375 & $\begin{array}{l}\text { High } \\
\text { strength }\end{array}$ \\
\hline S1b & $\begin{array}{c}\text { GBFS, } \\
357\end{array}$ & 1.34 & 3 & 0.420 & $\begin{array}{l}40 \% \text { sand } 0-4 \mathrm{~mm}, 60 \% \\
\text { gravel } 4-16 \mathrm{~mm} \text {, to meet } \\
\text { A/B } 16 \text { curve }\end{array}$ & 1.0 & 2364 & $\begin{array}{r}\text { Moderate } \\
\text { strength }\end{array}$ \\
\hline FA2 & FA, 425 & 16.5 & 5.9 & 0.223 & To meet $\mathrm{A} / \mathrm{B} 16$ curve & 3.0 & 2350 & $\begin{array}{l}\text { High } \\
\text { strength }\end{array}$ \\
\hline FA8 & FA, 425 & 16.5 & 5.9 & 0.253 & To meet A 16 curve & 3.0 & 2324 & $\begin{array}{r}\text { Moderate } \\
\text { strength }\end{array}$ \\
\hline MK1 & MK, 350 & 32.3 & 2.7 & 0.393 & To meet $\mathrm{A} / \mathrm{B} 16$ curve & 1.0 & 2186 & $\begin{array}{l}\text { High } \\
\text { strength }\end{array}$ \\
\hline
\end{tabular}

${ }^{a}$ Represented as $\mathrm{g} \mathrm{Na}_{2} \mathrm{Si}_{2} \mathrm{O}_{5} / 100 \mathrm{~g}$ precursor, where the solid component of sodium silicate solution of modulus 2.0 is given as $\mathrm{Na}_{2} \mathrm{Si}_{2} \mathrm{O}_{5}$. Where a different modulus of sodium silicate solution was used in some labs, the total activator dose was held constant but the ratio between silicate and hydroxide constituents was changed

${ }^{\mathrm{b}}$ Represented as g NaOH/100 g precursor

'Including water added within the aqueous activator solution, or separately from the activator, and with "binder" defined as the sum of precursor and solid activator components

${ }^{\mathrm{d}}$ Participants were instructed to match the A 16 (coarse) or A/B 16 (between coarse A and fine B) curves of DIN 1045-2 [3] as closely as possible; some labs could only access all-in aggregates or only two different aggregate fractions and this gave some intrinsic variability, whereas others were able to blend multiple fractions to give a closer match to the specified curve

${ }^{\mathrm{e}}$ The air content and density given here are nominal values used in mix design, and will vary depending on the nature of the aggregates, mixing and casting protocols used in each lab 


\section{Concrete mix design and curing}

The concrete mixes tested by TC members are summarised in Table 3, and the rationales for these mix designs are presented in the following sections. All concretes were designed to be cured under sealed conditions at ambient temperature $\left(20-25{ }^{\circ} \mathrm{C}\right.$ depending on local standard practice), with sufficient workability and appropriate setting times observed in preliminary testwork in selected (volunteer) laboratories, to enable mixing and casting to be achieved in the absence of proprietary admixtures. Care was taken in all laboratories to avoid contamination by (for example) adhered Portland cement-based materials in mixers and other equipment. Sealed curing was achieved by the use of polymeric films or bags according to established practice in each laboratory; samples were demoulded when sufficient strength had been gained to enable this to be done without damage to the samples, and the samples were then tightly wrapped to prevent moisture loss (again in polymeric films or bags) for continued sealed curing until testing.

\subsection{Slag-based concretes}

Two concrete mixes based on ground granulated blast furnace slag were designed, one for high performance and the other for moderate performance, denoted S3a and $\mathrm{S} 1 \mathrm{~b}$ respectively. Concrete mixes were designed and selected based on a campaign of mortar testing conducted by ASCEM B.V., with candidate mortars worked up into a small number of concrete mix designs submitted to the TC for the selection of two to take forward to the full test campaign. S3a was designed to reach a higher strength than S1b via its higher BFS content, higher activator dose and lower water/binder ratio. Both slag-based concretes had flow diameters of $300 \pm 10 \mathrm{~mm}$ determined according to EN 12350-5 [4].

While workable concretes at flow diameters of around $300 \mathrm{~mm}$ were reported in most laboratories, one participating laboratory experienced for mixes S3a and S1b an extremely harsh workability; neither mix could be appropriately compacted or cast. In such an instance, suspicion will obviously fall upon the aggregates used in that laboratory as the most likely cause of differences from other facilities, as each participant was using a locally available material. However, the aggregates used in those tests were a quarried siliceous (basaltic) aggregate and siliceous sand that have been found, and published, to give very good results for workability and strength in concretes based on both AAM and Portland cement binders. The laboratory was also using standard, well-established processes and equipment, and skilled personnel, to undertake the work. It is therefore unclear why these mixes could not be produced effectively in this one location.

\subsection{Fly ash-based concretes}

A full and detailed description of the mix design of the fly ash-based concretes, denoted FA2 (higher strength) and FA8 (moderate strength) respectively, is given in [5]. The paste formulations were designed by Curtin University by matching activator chemistry to the characteristics and composition of the amorphous, reactive component of the fly ash, based on the methodology of [6], resulting in a binder with $\mathrm{Si}$ / $\mathrm{Al}=3.0$ and $\mathrm{Na} / \mathrm{Al}=1.1$ in both concretes. These were then developed into mortar and concrete mixes by collaboration between Curtin University and BAM. FA2 was designed to reach a higher strength than FA8 via its lower water/binder ratio and through the selection of an intermediate (A/B 16 from DIN 1045-2 [3]; in between the defined curves A and B) aggregate grading curve rather than the coarser A 16 curve used in FA8. This came at the cost of a minor reduction in workability (although still producing a workable concrete); the EN 12350-5 [4] flow diameter of FA8 was $490 \mathrm{~mm}$ (F4 class of EN 206), but this was reduced to $370 \mathrm{~mm}$ (class F2) for FA2. Three laboratories reported the flow diameters for these concretes, and determined values which were in agreement to within $\pm 50 \mathrm{~mm}$ in each case.

Several laboratories reported setting times of more than 1 day for the fly ash-based mixes, while conversely, one laboratory reported flash setting that made the compaction and casting of specimens very difficult. The staff of this laboratory reported that care had been taken to avoid any chance of contamination by Portland cement or other rapidly-reacting materials, and the mixers used were not reported to be of unusual power or characteristics, so the reason for this behaviour is unknown. 


\subsection{Flash-calcined metakaolin based concretes}

A full description of the mix design protocols applied for concretes based on flash-calcined metakaolin is given in [7]; the paste and concrete formulations were developed at the Université de Toulouse as an adaptation of the mix denoted C7 in [7]. This mix had the highest compressive strength among a wide spread of mixes tested in that work, while also showing a moderate slump (consistency class S3/S4 [7] according to EN 206 [8]) and an EN 12350-5 flow diameter of $530 \pm 60 \mathrm{~mm}$, and it has been validated through larger-scale testing including the production of reinforced beams using batch sizes exceeding $140 \mathrm{~L}$ [7]. A single metakaolin-based mix was used throughout the testing campaign.

\subsection{Testing methodology}

The concretes defined in Table 3 were produced in fifteen participating laboratories. All laboratories were instructed to use established expertise, facilities and procedures to produce concretes according to these common mix designs. Therefore, each laboratory generally followed a local modification of the mixing, casting and curing procedures described in the relevant ASTM, EN, AS or CSA standards; none of these standards describe precisely a procedure which is suitable for the production and testing of alkali-activated concretes, so each laboratory adapted their standard method to apply a procedure which is suited to its own facilities. All participating laboratories have extensive expertise in the production and testing of alkali-activated concretes, and used fullytrained personnel (technical or postdoctoral staff, or experienced graduate students) to apply the agreed procedures.

Compressive strength testing was conducted according to either ASTM C39 [9] or EN 12390-3 [10]; where $150 \mathrm{~mm}$ cylindrical samples were used under either protocol, the results were multiplied by 1.25 to convert to equivalent cube strengths, mirroring the factor used for equivalency in strength classes in EN 206 [8]. Following the guidance of BS 8500-1 [11] which considers $150 \mathrm{~mm}$ and $100 \mathrm{~mm}$ cubes to give equal strengths, no further correction was applied to account for sample size.
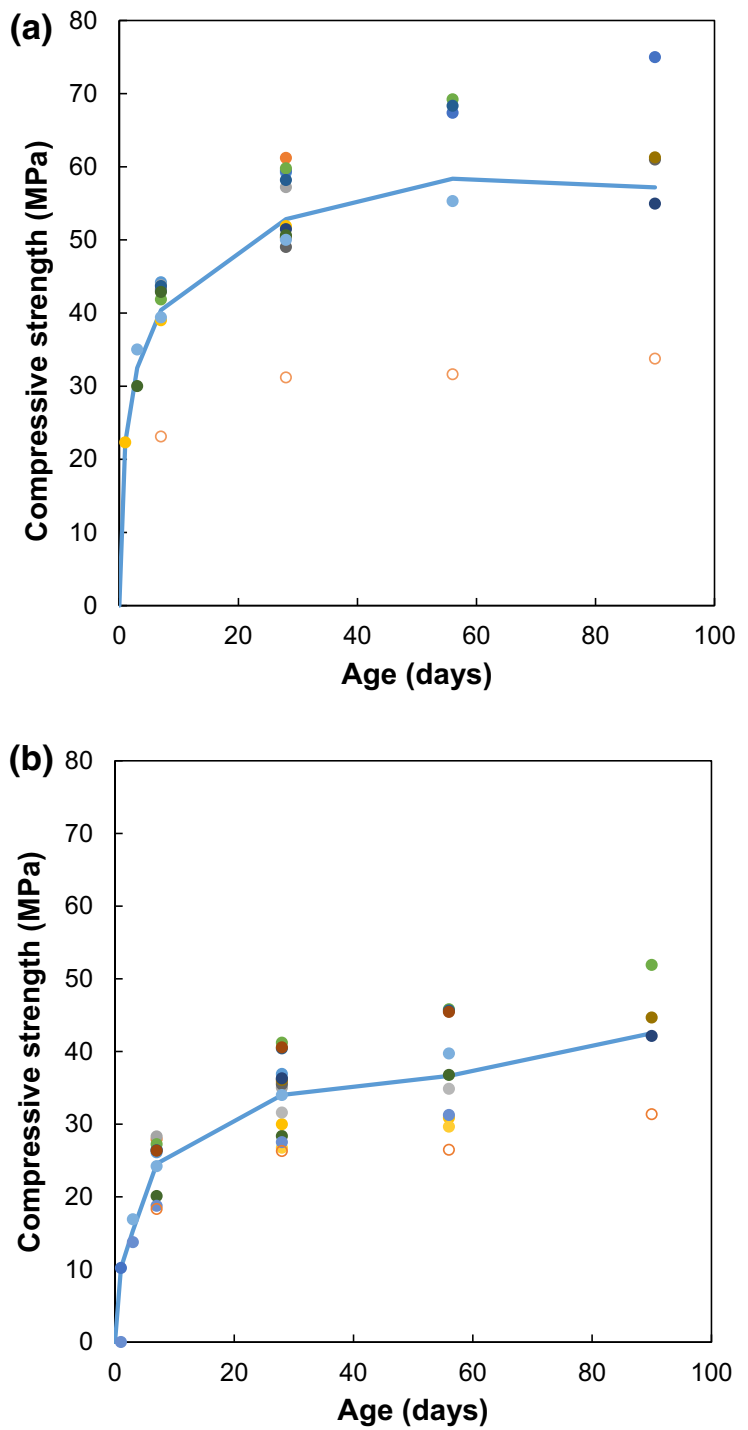

Fig. 1 Compressive strengths determined for slag-based AAM concretes: a S3a, and b S1b. All samples were cured under sealed conditions at $20-25{ }^{\circ} \mathrm{C}$, except the hollow orange circles in each plot, which represent the laboratory that cured samples underwater. Each point represents a single reported result; each laboratory returned between 1 and 6 strength result(s) per age, and not all laboratories returned results for each mix at every age. The solid line represents the mean of all reported data

\section{Results and discussion}

\subsection{Compressive strength—slag-based AAM concretes}

The compressive strength results obtained for the two slag-based concrete mixes are presented in Fig. 1. 
The data in Fig. 1 show that the higher activator dose and BFS content, and lower water content, of S3a resulted in an effective increase in the compressive strength. One laboratory reported a very low set of strength data for S3a, which may be attributed to their use of underwater (rather than sealed) curing conditions causing alkali washout. However, the same laboratory reported strengths for $\mathrm{S} 1 \mathrm{~b}$ (also cured underwater) that were in relatively good agreement with the results obtained in other laboratories, falling somewhat below the mean but not so evidently discrepant. This provides an initial indication that the sensitivity of these AAM concretes to differences in mixing and curing parameters may depend on factors that are not generally considered in concrete technology; the concrete with a higher activator dose appears to have lost the advantage of that extra activator (and has probably actually lost some of the extra activator through washout) due to having been immersed when it was demoulded $24 \mathrm{~h}$ after casting. The two slag-based concretes cast in that particular laboratory had similar strengths at later ages despite the formulation difference.

The mean strengths of both mixes increased consistently up to 56 days; there is an apparent slight decrease in strength in the S3a data at 90 days, but this can be attributed to an artefact in the calculation of the mean in the combined data set, as one of the laboratories which reported high strengths at 56 days did not return 90-day data. No participating laboratory observed any strength regression over time in any of the concretes studied, regardless of precursor nature or mix design, and consistent with a large body of literature on well-formulated alkali-activated pastes, mortars and concretes cured under appropriate conditions.

Figure 1a also shows some evident clustering of results into a set that are very close to the mean, and another set that are significantly higher. This clustering is related to the very good intra-laboratory reproducibility of the test results reported; for each batch of samples cast in each laboratory, there was no more than $2 \mathrm{MPa}$ difference between the highest and lowest strength results recorded. One laboratory reporting multiple batches did have a higher deviation than this, with around $9 \mathrm{MPa}$ difference between the means of the two batches reported, but the reason for this difference is not clear. Further discussion of potential causes of between-batch variation are given below in the context of fly ash-based AAM concretes. Both the intra-laboratory and inter-laboratory variability in compressive strength data are also generally consistent with the results of a recent round-robin test of plain Portland cement concretes [12]. In that study, 15 participating laboratories returned low intra-laboratory deviations in 28-day compressive strength, but with the highest returned value being $\sim 50 \%$ higher than the lowest, even using identical fine and coarse aggregates, and with highly standardised mixing and casting protocols being applied in all laboratorieswhich was not the case in the present study. So, based on this comparison the results in Fig. 1 can be taken to show that alkali-activated slag concretes are not any more variable in compressive strength, in an interlaboratory comparison, than are plain Portland cement concretes.

Comparing Fig. 1a and b, it appears that the higherstrength mix, S3a, shows a smaller scatter in the results than S1b, other than for the discrepant case of S3a discussed above; considering the 28-day data for both mixes, there is approximately a $20 \%$ difference between the highest and lowest valid (non-outlier) data points for S3a, but around 30\% for S1b. Figure 2 shows the standard deviations associated with the compressive strengths of each of the five concrete mixes as a function of age. The percentage standard deviation of the data for S3a is also clearly lower than

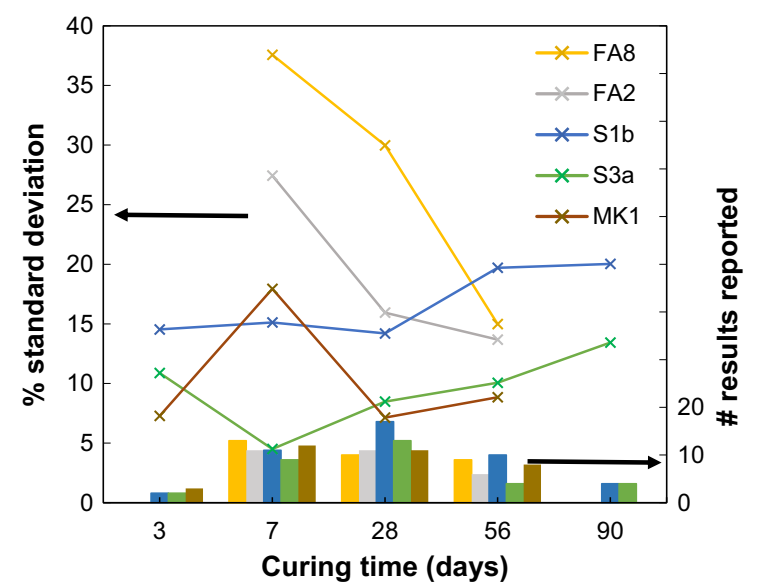

Fig. 2 Percentage standard deviations in reported compressive strength data (excluding evident outliers) as a function of concrete mix and curing time (curves, left-hand vertical axis), and the number of results contributing to each of these standard deviations (columns, right-hand vertical axis). Lines linking the data points are to guide the eye 


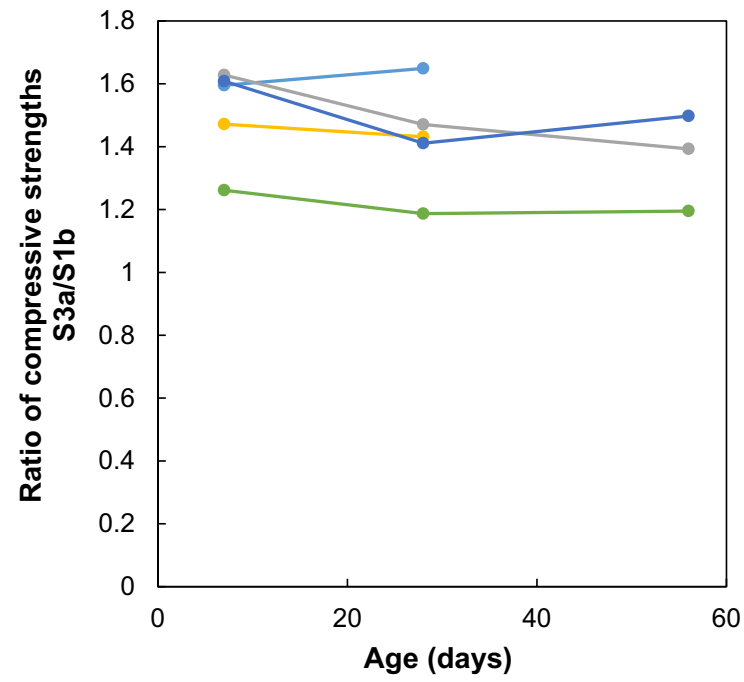

Fig. 3 Ratio of mean compressive strengths within each laboratory between S3a and S1b at each age, for laboratories that returned valid results for both concretes. Each line represents one laboratory

for S1b at each age. However, the slag mixes show significantly lower standard deviations than the fly ash mixes at each age; the reasons for the high variability in the fly ash concrete strengths will be explored in more detail in the following section.

Figure 3 shows the ratios between the mean compressive strengths reported for S3a and for S1b by each laboratory that returned valid results for both mixes. These data show that the participating laboratories achieved a remarkable consistency in the ratio between the strengths of the two mixes (which differ very significantly in performance levels), and thus highlighting that the scatter in Fig. 1 is likely to be related to the characteristics of the aggregates used in each laboratory, and the influence of slight variations in laboratory infrastructure (e.g. mixer type and power consumption, compaction energy, mould type). This further demonstrates that well-operated concrete laboratories in various parts of the world can reproducibly produce alkali-activated concretes, and that the relative performance levels of these concretes are highly comparable between laboratories even if the absolute performance levels differ.

The compressive strength data presented here thus provide significant insight into the relative performance and robustness of alkali-activated slag concrete mix designs:
- For both mixes, the differences between the highest and lowest valid results obtained increased with curing duration. Some laboratories observed that strength increase essentially finished at 28 days in $\mathrm{S} 1 \mathrm{~b}$, but others found that the strength gain continued beyond this time, and S3a generally showed continued strength gain.

- Higher activator and BFS contents, and lower water/binder ratio, resulted in higher strengths for S3a than S1b.

- A mix with a low activator content (e.g. S1b) may be considered less robust in terms of on-going strength evolution. It is using its activator efficiently to generate moderate strength, but when the activator is largely consumed (e.g. around 28 days), the strength development is prone to slow down or cease. This is likely to be related to a reduction in the available alkalinity (effectively, the $\mathrm{pH}$ ) in the pore solution as the alkali activator is consumed, leaving a long-term process which is more akin to slag hydration (which is slow) rather than continued alkali-activation. However, this is variable between laboratories and the factors causing this variability are not well understood, and cannot be readily explained from the analysis provided here, but do lead to a broader spread of strength data at later age.

- A mix with a high activator content (e.g. S3a) is more robust, because even if the available activator is not used with full effectiveness, there is sufficient excess that strength increase continues up to at least 90 days; a 7-day strength of around 30-35 MPa increases to 50-60 MPa at 28 days, and 55-75 MPa at 90 days. However, this mix is still prone to issues with washout as were noted above; underwater curing returned the material to a lower performance level, similar to that of S1b.

- The parameter selected for controlling the concrete strengths of S3a and S1b was the activator dose, which is more influential at early age due to the kinetic effects of high $\mathrm{pH}$ in accelerating slag reaction [13]. Thus, the difference in strength between the two mixes, shown in Fig. 3, decreased at increasing age of the concretes in almost every laboratory which reported results for both mixes. The lower activator dose causes a greater performance penalty at early age, but this is less critical at later age (with the provision of an appropriate 

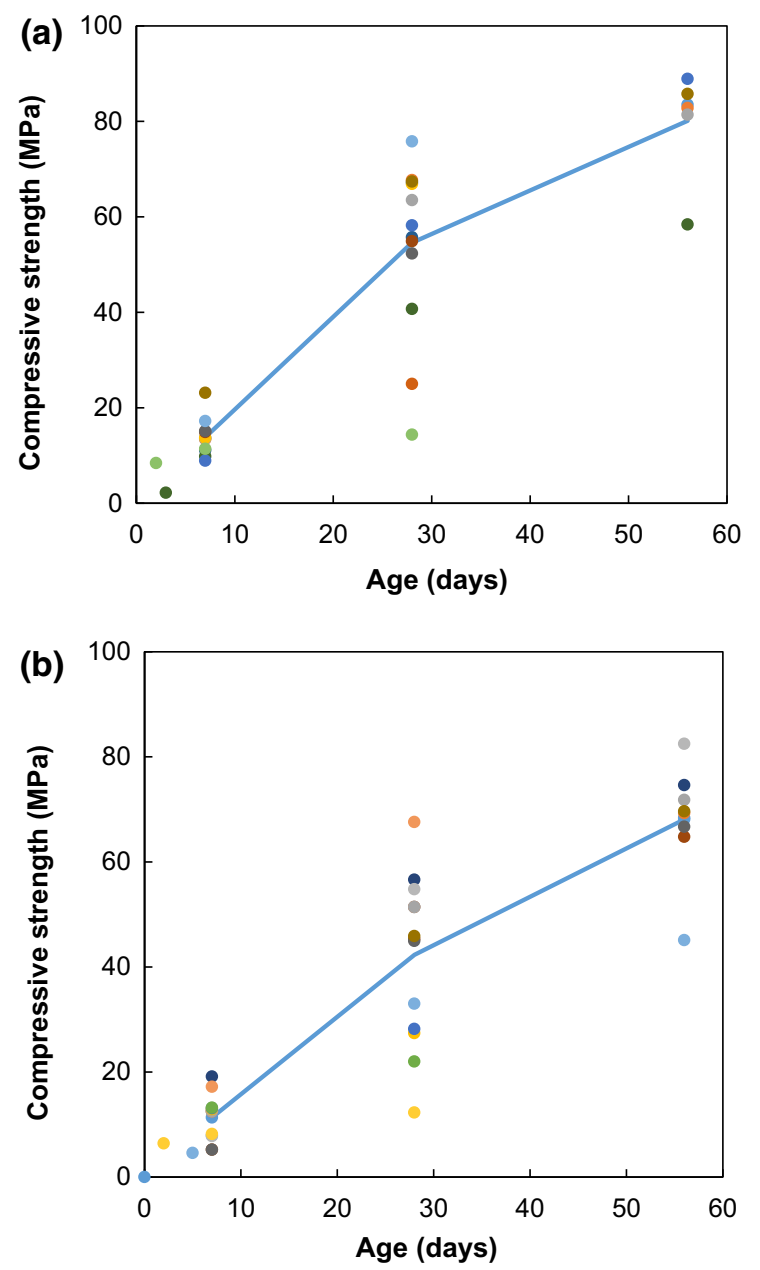

Fig. 4 Compressive strengths determined for fly ash-based AAM concretes cured under sealed conditions at $20-25{ }^{\circ} \mathrm{C}$ : a FA2, and b FA8. Each point represents a single reported result; each laboratory returned between 1 and 6 strength result(s) per age; not all laboratories returned results for each mix at every age. The solid line represents the mean of all reported data

curing environment) as the microstructures of both concretes continued to evolve.

\subsection{Compressive strength—fly ash-based AAM concretes}

The compressive strength results obtained for the two fly ash-based concrete mixes are presented in Fig. 4.

The data in Fig. 4 show that the addition of extra water in FA8, along with the change in aggregate grading, gave a decrease in compressive strength at all ages compared to FA2. However, it is striking that there is an enormous spread in compressive strength values for both fly ash-based concretes in Fig. 4, particularly at 28 days (although this is to some degree exacerbated by the fact that this is the age at which the largest number of data points were returned, Fig. 2). The laboratories reporting the lowest 28-day strengths for both FA2 and FA8 did not continue testing up to 56 days, but there were also laboratories achieving higher 28-day strengths that did not return 56-day data, so this cannot be the sole reason for the reduced spread in data (and correspondingly the reduced standard deviation, Fig. 2).

Additional key aspects to note from the data presented in Fig. 4 include:

- Curing was conducted at ambient temperature (between 20 and $25^{\circ} \mathrm{C}$ depending on local standard practice), under sealed conditions, in all cases except one laboratory. That laboratory used sealed curing boxes with liquid water in the bottom to maintain a high humidity. This yielded 7-day strengths below the overall average, but 28- and 56-day strengths matching or exceeding the average, potentially due to some early drying of the samples. Nonetheless, the development of satisfactory 7-day strengths under ambient temperature conditions, and very good 28-day strengths, provides conclusive evidence that heat-curing is not required for a well-designed alkali-activated fly ash concrete that is based on a relatively good quality fly ash combined with a suitable activator; various assertions to the contrary within the academic literature are not necessarily correct in the general sense.

- The gain in strength from 28 to 56 days in all laboratories is much greater than was observed for slag-based binders, or for conventional cements. The rate of increase of strength is only slightly lower from 28 to 56 days than it is from 7 to 28 days, when considering the mean values among all reported data. Within the data reported from individual laboratories, the rate of increase is slightly lower than this because, as was noted above, several laboratories that reported relatively low 28-day strengths did not report 56-day strengths. However, the high degree of longerterm on-going strength development does raise important questions about the most relevant testing age for both characteristic strength and durability 
of fly ash-based AAMs. The standard age of 28 days that is almost universally used for compressive strength (and other) tests of Portland cement-based materials was originally selected in the second half of the nineteenth century (see [14] and literature cited therein), and it is widely assumed to be an age at which the ultimate strength and other maturity characteristics of the material are achieved or approached. However, modern Portland cements gain a much higher percentage of their ultimate strength by 28 days than do the alkali-activated fly ash concretes tested here. It is likely that the application of elevated-temperature curing would lead to a higher 28-day/56-day strength ratio, but the testing programme reported here was designed to investigate solely roomtemperature cured materials, so this was not tested.

- Related to the preceding point, the 3- and 7-day strengths of the fly ash-based AAM concretes were low in many laboratories, although some results between 15 and $20 \mathrm{MPa}$ were reported for both FA2 and FA8 at 7 days.

- One laboratory which produced multiple batches of concrete, under very detailed quality control and with otherwise excellent reproducibility, found that one batch had identical 7-day but much lower (by 40\%) 28-day strengths than the others. The only potential explanation that could be reached

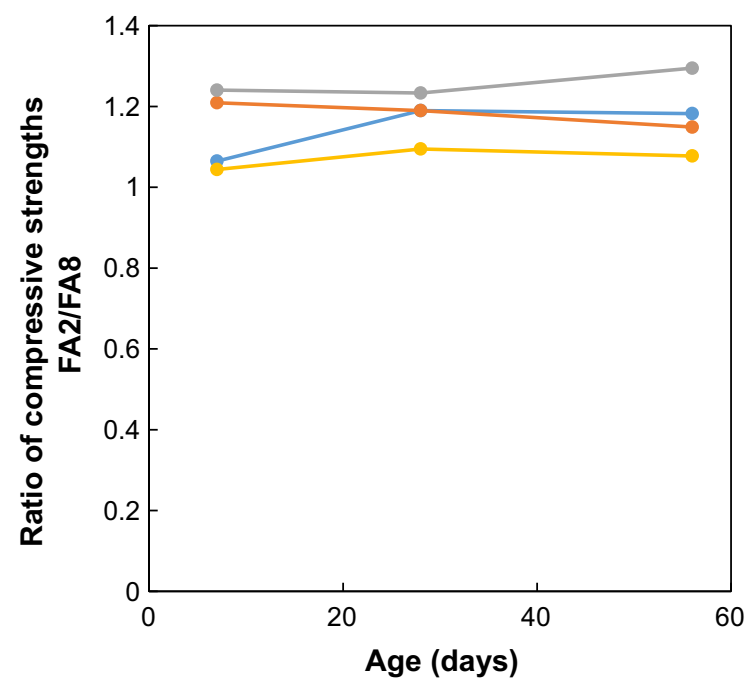

Fig. 5 Ratio of mean compressive strengths within each laboratory between FA2 and FA8 at each age, for laboratories that returned valid results for both concretes. Each line represents one laboratory for this result was that all batches had been cured under sealed conditions in plastic bags, and slightly thinner bags were used for this particular batch. It is not possible to state definitively that this would be sufficient to cause such a difference in performance - and such sensitivity to minor variations in curing environment has not previously been reported in the open literature for fly ash-based AAMs - so this must be considered at best a speculative explanation for the observed results.

Figure 5 presents the fly ash concrete data in a form comparable to Fig. 3, showing the ratios between the strengths of the two mixes in each individual participating laboratory.

Figure 5 shows that the $\sim 10 \%$ difference in water/binder ratio, as well as the improved aggregate grading curve, yielded a strength for FA2 that is consistently around $20 \%$ higher than FA8 in a withinlab comparison. The within-lab comparisons show more clearly the difference between the two mixes that are less obvious from Fig. 4 due to the scatter within each inter-laboratory data set. The ratio between the concrete strengths is remarkably consistent as a function of time in each participating laboratory. The use of water-binder ratio and aggregate grading as the key parameters to change the concrete strength for the fly ash-based concretes, in contrast to the use of the

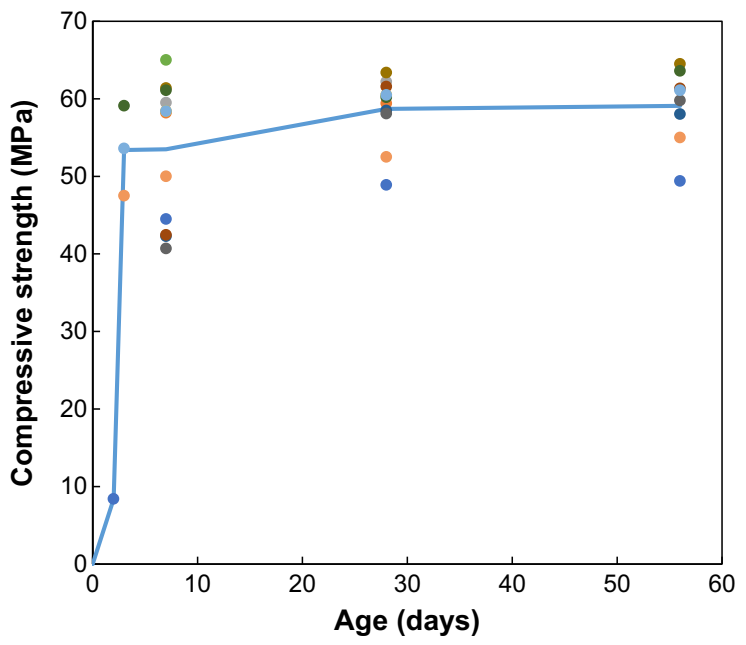

Fig. 6 Compressive strengths determined for metakaolinbased AAM concretes cured under sealed conditions at 20-25 ${ }^{\circ} \mathrm{C}$. Each point represents a single reported result; each laboratory returned between 1 and 6 strength result(s) per age; not all laboratories returned results at every age. The solid line represents the mean of all reported data 
activator dose to control the performance of the slagbased concretes (Fig. 3), did not lead to a strong timedependence in the strength ratio as was identified above for slag-based AAMs.

\subsection{Compressive strength—metakaolin-based AAM concretes}

Figure 6 shows the compressive strengths of the metakaolin-based AAM concretes tested in each laboratory, as a function of age. The scatter in these data is smaller than for the fly ash or slag-based concretes, particularly at later age, and there are no evident outliers at any age. The metakaolin-based AAM concretes were, in all except one laboratory, within $5 \mathrm{MPa}$ of their ultimate (28- or 56-day) strength by 7 days. The small number of 3-day data returned were also high, within $10 \mathrm{MPa}$ of the ultimate strengths that were reached in those laboratories, although one laboratory did report a strength of less than $0.3 \mathrm{MPa}$ at 5 days (but still exceeded $40 \mathrm{MPa}$ at 7 days). This is a striking difference from the slagbased and fly ash-based AAM concretes, which gained strength much more slowly, although the activator dose used in the metakaolin-based mixes was much higher than in the other concretes. Highlighting once more that these specimens were all cured at ambient temperature, this set of results provides a definitive answer to questions around whether all AAMs require heat-curing for satisfactory early strength generation; it is eminently clear that this is not the case.

These data also show no evidence of strength regression or other forms of sample 'misbehaviour' that are sometimes described in the technical literature for clay-based AAMs. As for the fly ash-based and slag-based binders, the within-laboratory reproducibility of the test results was very good, with no more than $10 \%$ spread across replicates in any given laboratory. Comparing different laboratories, there is not a strong correlation between those which achieved relatively higher (or lower) strengths in the metakaolin-based mixes compared to the fly ash-based mixes; for example, one laboratory which obtained low strengths for both fly ash-based concretes returned an above-average strength for the metakaolin-based mix. The laboratory which cured in boxes over liquid water again reported a 7-day strength below the mean, but values were comparable to the other laboratories at 28 and 56 days. It is therefore likely that the differences are attributable, at least in part, to differences in compatibility between binders and aggregates in each laboratory (probably depending on aggregate mineralogy and geometry), rather than being systematically attributable to any particular aspect of laboratory practice.

\section{General remarks and connected studies}

The results presented here show that the basic mechanics of concrete strength testing as specified in existing standards for Portland cement concrete appear to be applicable for alkali-activated concretes. By means of compressive strength testing, the difference between mix designs is identifiable for each precursor. The two performance levels of concrete that were produced for each of the slag and fly ash precursors give a useful starting point for the comparison of concrete durability. However, the use of 28 days as a testing age to determine the 'ultimate' properties is not necessarily appropriate for all of these materials. The flash calcined metakaolin-based mixes can reach their ultimate strength much sooner than this, and so waiting until 28 days is not a good time investment. Conversely, the fly ash-based concretes continue to gain much more strength beyond this age, so could benefit from 56-day testing, as has been discussed extensively for other materials such as Portland cement blended with slower-reacting pozzolans. Among the three sets of concretes tested, those based on slag are probably the closest to being wellrepresented by a 28 -day determination of characteristic strength.

Additional work reporting material parameters and characterisation of these concretes (and their constituent pastes and mortars), including permeability, porosimetry and more detailed microstructural analysis related to durability, has been published by various participating groups in publications including [5, 13, 15-20]. In two papers that will follow this publication, round-robin test results for chloride migration/diffusion, carbonation, alkali-silica reaction, sulfate and freeze-thaw/frost-salt resistance, will be presented and discussed. 


\section{Conclusions}

This paper has reported the compressive strength results collected through the round-robin testing of RILEM Technical Committee 247-DTA, for alkaliactivated concretes based on ground granulated blast furnace slag, on fly ash, and on flash-calcined metakaolin. Two mixes were formulated for the slag and the fly ash precursors, and one for the metakaolin; where two mixes were used per precursor, these were designed to show distinguishable performance levels, and the expected differences were observed across laboratories. The round-robin test outcomes showed that each participating laboratory was able to reproducibly produce alkali-activated concretes, with interlaboratory deviations that are broadly consistent with expectations for Portland cement-based concretes. The differences in compressive strength between each pair of concretes (where two concretes were generated from a single precursor) were very consistent between participating laboratories; the ratios of the strengths of the two slag-based and the two fly ash-based concretes provided a very good indication of reproducibility of the test results. The use of activator dose as a parameter to manipulate concrete strength leads to a strong time dependency in the ratio between concretes of differing performance levels, but this is not evident when the water/binder ratio is used to manipulate concrete strength. The concretes characterised and tested in this study appear suitable, in terms of basic mechanical properties, to be carried forward into the durability testing programme of the RILEM TC 247-DTA round robin tests.

Acknowledgements The authors would like to thank all members of RILEM TC 247-DTA for the valuable discussions in planning the activities for the round robin. Particularly we would like to acknowledge the immense contribution of A. Buchwald (ASCEM, Netherlands), W. Rickard and A. van Riessen (Curtin University, Australia), G. Gluth (BAM, Germany), R. Pouhet and M. Cyr (Toulouse University, France) in developing the concrete mix designs evaluated; and for arranging the donations of raw materials, and the logistics for their distribution to all the round robin participants. The participation of Dr Andrew Dunster (BRE, United Kingdom) in the round-robin testing and enriching the associated discussions is very gratefully acknowledged. Special thanks are also due to Ecocem (France), BauMineral (Germany) and Argeco (France) for the generous donation of several tonnes of slag, fly ash and flash-calcined metakaolin to the members of this technical committee; and to PQ Corporation and Grupo IQE for the donation of activator constituents to some of the participating laboratories.

Funding The participation of J. L. Provis and S. A. Bernal (at U. Sheffield), S. Nanukuttan and D. Bondar (at Queen's University) in this research was sponsored by the Engineering and Physical Sciences Research Council (EPSRC; UK) under Grant Number EP/M003272/1. Participation of V. Ducman was financially supported by the Slovenian Research Agency Programme Group P2-0273. The work and research of K. Dombrowski-Daube in RILEM TC-247 DTA was supported by ZIM - Central Innovation Program, German Federal Ministry of Economic Affairs and Energy (BMWi) by order of the German Bundestag. The contribution of the team at TU Delft led by G. Ye was supported by the Materials innovation institute M2i/ Netherlands Organisation for Scientific Research (STW/M2i Project 13361). The contributions of K. Arbi were also supported by Delta Concrete Consult BV.

\section{Compliance with ethical standards}

Conflict of interest The authors declare that they have no conflict of interest.

Open Access This article is distributed under the terms of the Creative Commons Attribution 4.0 International License (http:// creativecommons.org/licenses/by/4.0/), which permits unrestricted use, distribution, and reproduction in any medium, provided you give appropriate credit to the original author(s) and the source, provide a link to the Creative Commons license, and indicate if changes were made.

\section{References}

1. Provis JL, van Deventer JSJ (eds) (2014) Alkali-activated materials: state-of-the-art report, RILEM TC 224-AAM. Springer/RILEM, Dordrecht

2. Beushausen H, Fernández Luco L (2015) Performancebased specifications and control of concrete durabilitystate of the art report, RILEM TC 230-DUC. Springer/ RILEM, Dordrecht

3. Deutsches Institut für Normung (2008) Tragwerke aus Beton, Stahlbeton und Spannbeton - Teil 2: Beton Festlegung, Eigenschaften, Herstellung und Konformität Anwendungsregeln zu DIN EN 206-1 (DIN 1045-2:2008). Berlin, Germany

4. European Committee for Standardization (CEN) (2009) Testing fresh concrete. Flow table test (EN 12350-5), Brussels, Belgium

5. Gluth GJG, Rickard W (2015) Design and characterization of fly ash-based geopolymer concretes for a round-robin durability testing program. In: Leonelli C, Romagnoli M (eds) Geopolymers: the route to eliminate waste and emissions in ceramic and cement manufacturing. Hernstein, Austria, Engineering Conferences International/Società Ceramica Italia, pp 67-70 
6. Williams RP, van Riessen A (2010) Determination of the reactive component of fly ashes for geopolymer production using XRF and XRD. Fuel 89(12):3683-3692

7. Pouhet R, Cyr M (2016) Formulation and performance of flash metakaolin geopolymer concretes. Constr Build Mater 120:150-160

8. European Committee for Standardization (CEN) (2013) Concrete: specification, performance, production and conformity (EN 206:2013), Brussels, Belgium

9. ASTM International (2018) Standard test method for compressive strength of cylindrical concrete specimens (ASTM C39/C39 M - 18), West Conshohocken, PA

10. European Committee for Standardization (CEN) (2009) Testing hardened concrete. Compressive strength of test specimens (EN 12390-3), Brussels, Belgium

11. British Standards Institution (2015) Concrete-Complementary British Standard to BS EN 206-1-Part 1: method of specifying and guidance for the specifier (BS 8500-1), London, UK

12. Bokan Bosiljkov V, Kramar Fijavž M, Serdar M (2018) Mechanical properties of cement based materials-extended round robin test of COST Action TU 1404. In: Azenha M, Schlicke D, Benboudjema F, Jedrzejewska A, (eds) Proceedings of SynerCrete'18, International conference on interdisciplinary approaches for cement-based materials and structural concrete. Funchal, Portugal, RILEM proceedings PRO121, pp 47-54

13. Bondar D, Ma Q, Soutsos M, Basheer M, Provis JL, Nanukuttan S (2018) Alkali activated slag concretes designed for a desired slump, strength and chloride diffusivity. Constr Build Mater 190:191-199

14. Gonnerman HF (1958) Development of cement performance tests and requirements (Research Department Bulletin \#93). Portland Cement Association, Chicago
15. Puertas F, González-Fonteboa B, González-Taboada I, Alonso MM, Torres-Carrasco M, Rojo G, Martínez-Abella F (2018) Alkali-activated slag concrete: fresh and hardened behaviour. Cem Concr Compos 85:22-31

16. Dehghan A, Peterson K, Riehm G, Herzog Bromerchenkel L (2017) Application of X-ray microfluorescence for the determination of chloride diffusion coefficients in concrete chloride penetration experiments. Constr Build Mater 148:85-95

17. Kramar S, Šajna A, Ducman V (2016) Assessment of alkali activated mortars based on different precursors with regard to their suitability for concrete repair. Constr Build Mater 124:937-944

18. Ducman V, Kramar S, Šajna A (2018) Alkali activated repair mortars based on different precursors (Ch.11). In: Pacheco-Torgal F, Melchers RE, Shi X, de Belie N, Van Tittelboom K, Sáez A (eds) Eco-efficient repair and rehabilitation of concrete infrastructures. Woodhead Publishing, Cambridge, pp 263-292

19. Noushini A, Castel A (2018) Performance-based criteria to assess the suitability of geopolymer concrete in marine environments using modified ASTM C1202 and ASTM C1556 methods. Mater Struct 51(6):146

20. Yao W, Shi Y, Xia K, Peterson K (2019) Dynamic fracture behavior of alkali-activated mortars: effects of composition, curing time and loading rate. Eng Fract Mech 208:119-130

Publisher's Note Springer Nature remains neutral with regard to jurisdictional claims in published maps and institutional affiliations. 